\title{
JWST telescope integration and test progress
}

Gary W. Matthewsa, Tony L. Whitmana, Lee D. Feinberg ${ }^{b}$, Mark F.

Voyton $^{b}$, Juli A. Lander ${ }^{b}$, Ritva Keski-Kuhab

a - Harris Corporation

b - NASA Goddard Space Flight Center

SPIE XXXX-XX

June 2016 


\section{OTIS AI\&T Status}

\section{- Telescope Test Activities}

- Optical Telescope Element (OTE) and OTE/ISIM (OTIS) integration will occur at GSFC in the large SSDIF clean room

- Most of OTE Optical Ground Support Equipment (OGSE) has been completed and was used for Pathfinder integration operations

\section{- Pathfinder OGSE Test Program}

- A series of three cryo tests planned prior to the flight that increase in complexity and designed to cover all aspects of the flight test program

- CCT - Chamber Commissioning with the OGSE installed - Complete

- OGSE\#1 - Center of Curvature and Dynamic Testing - Complete

- OGSE\#2 - Half Pass and Pass and a Half Testing

- Thermal Pathfinder (TPF) - OTIS Thermal Simulation

- Optical Telescope Element (OTE) Integration

- Optical integration of the flight OTE scheduled for Fall 2015 


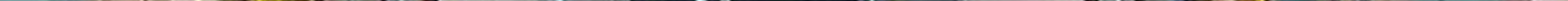




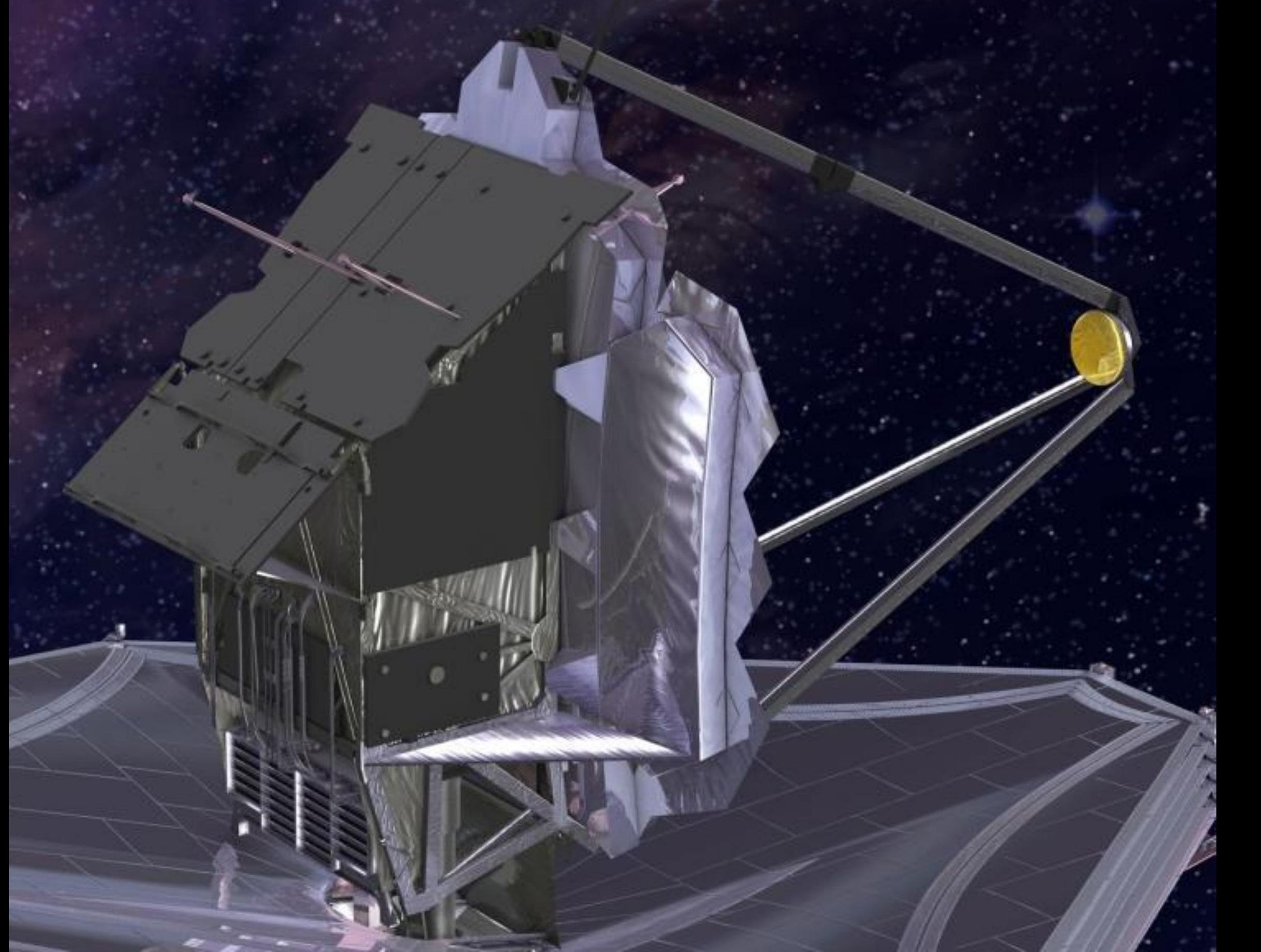




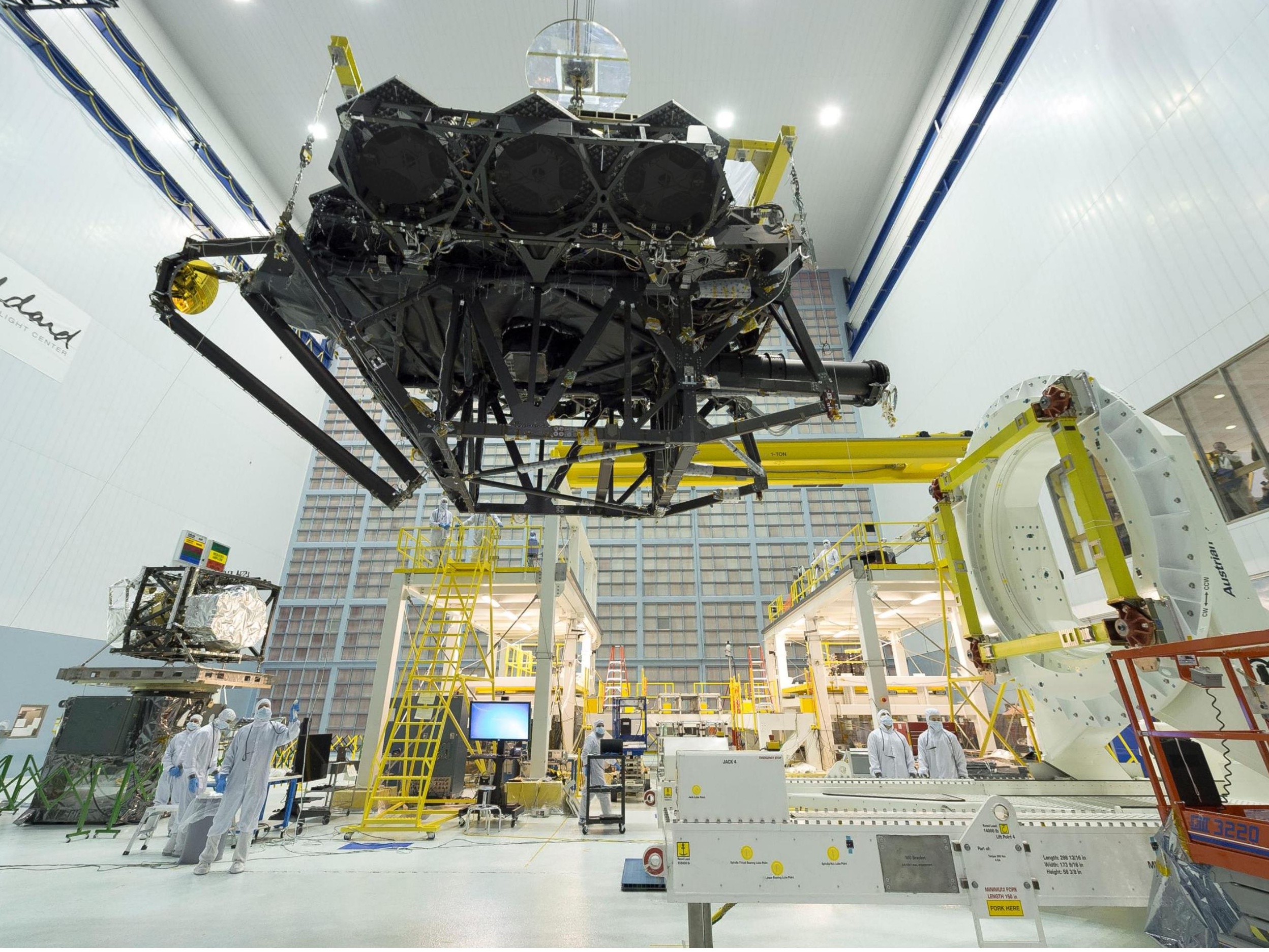




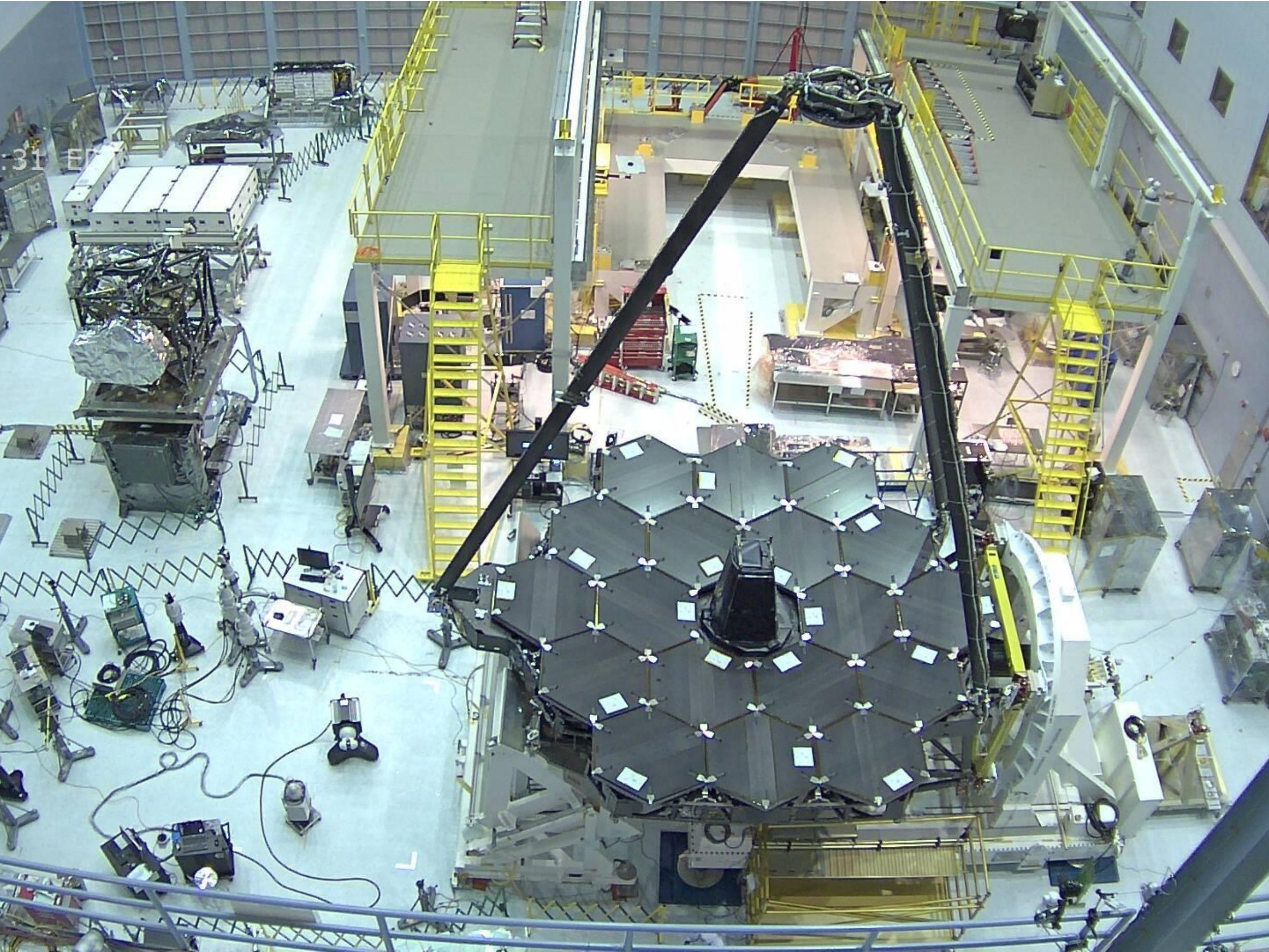




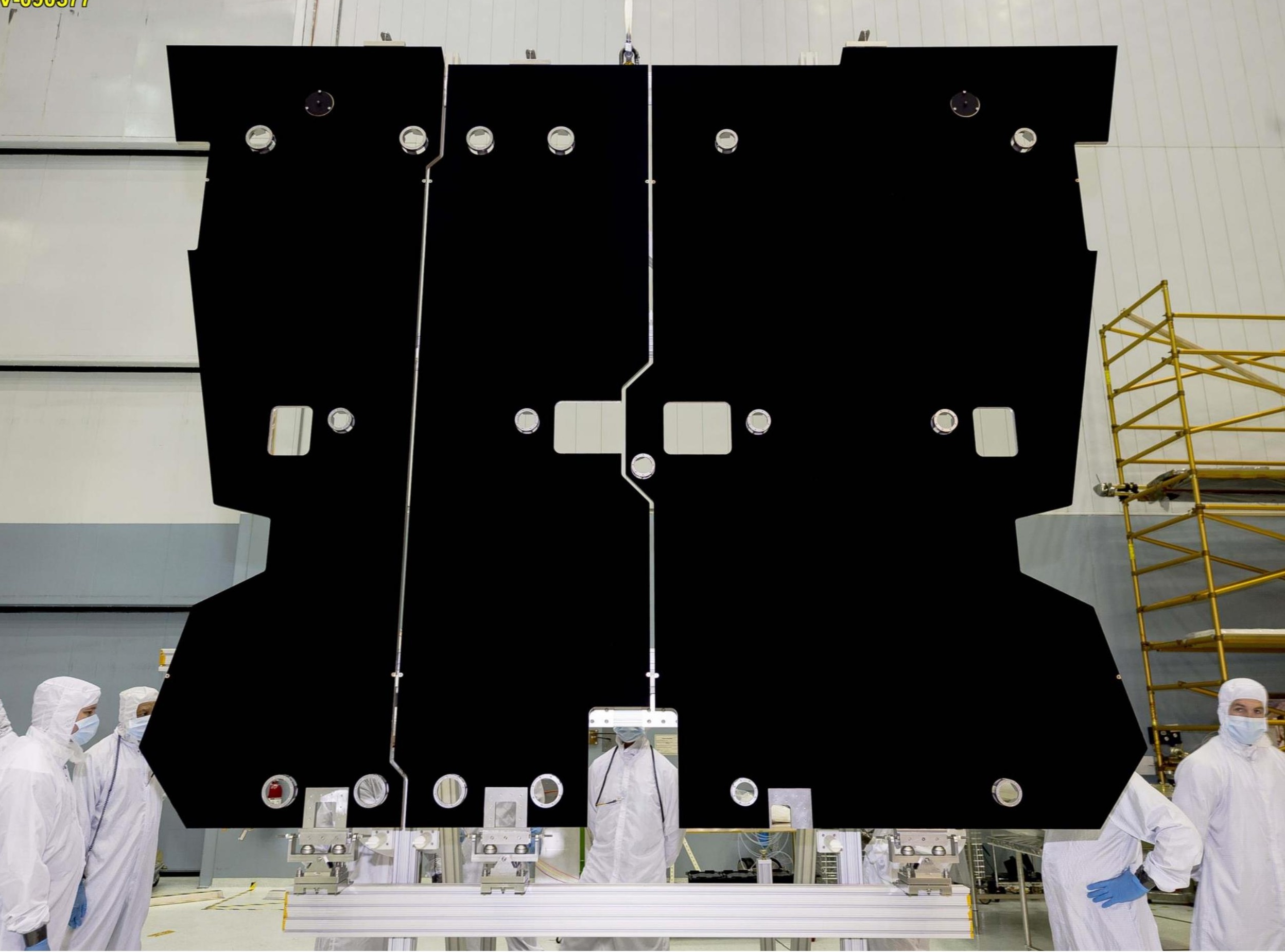




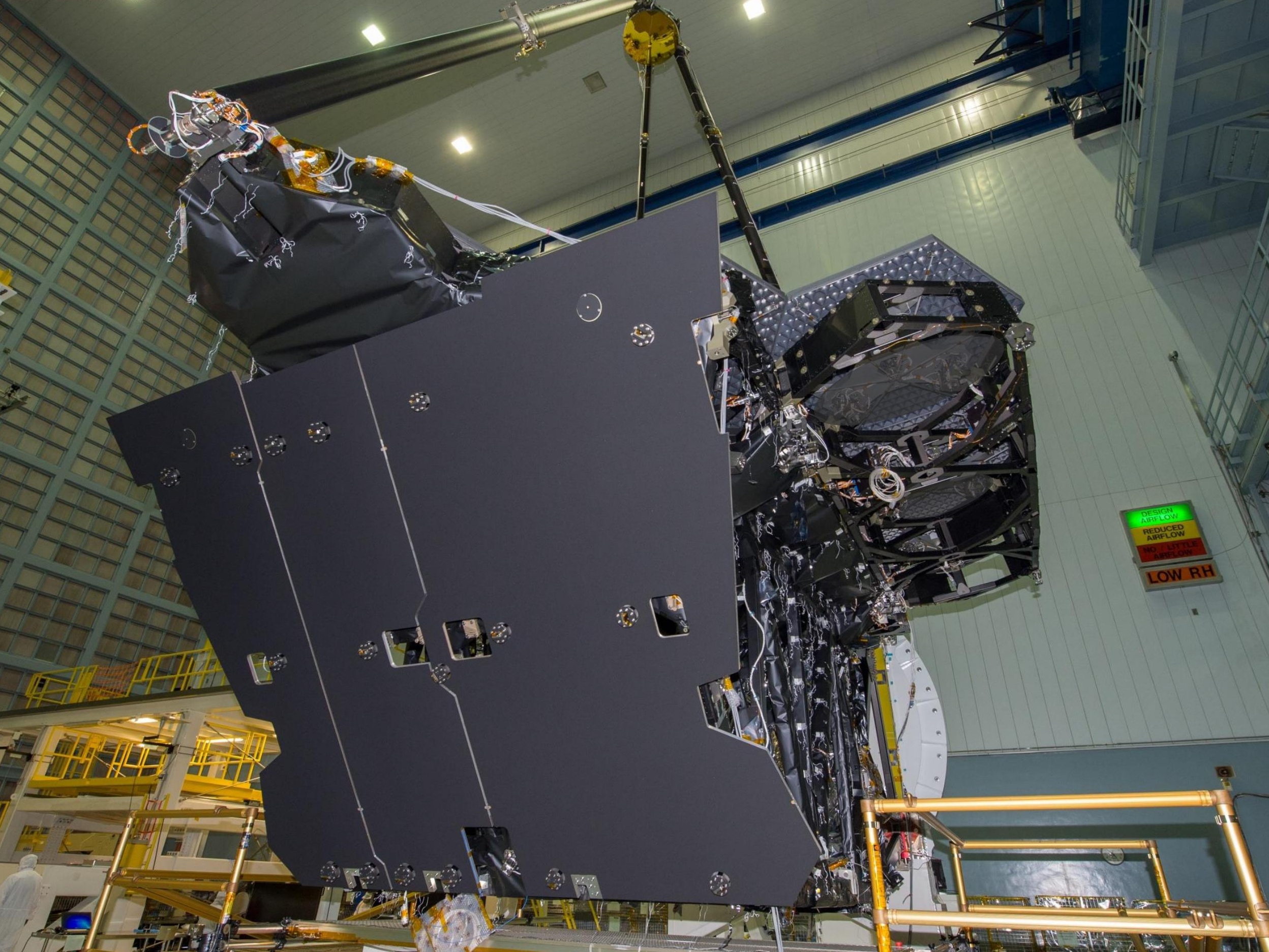




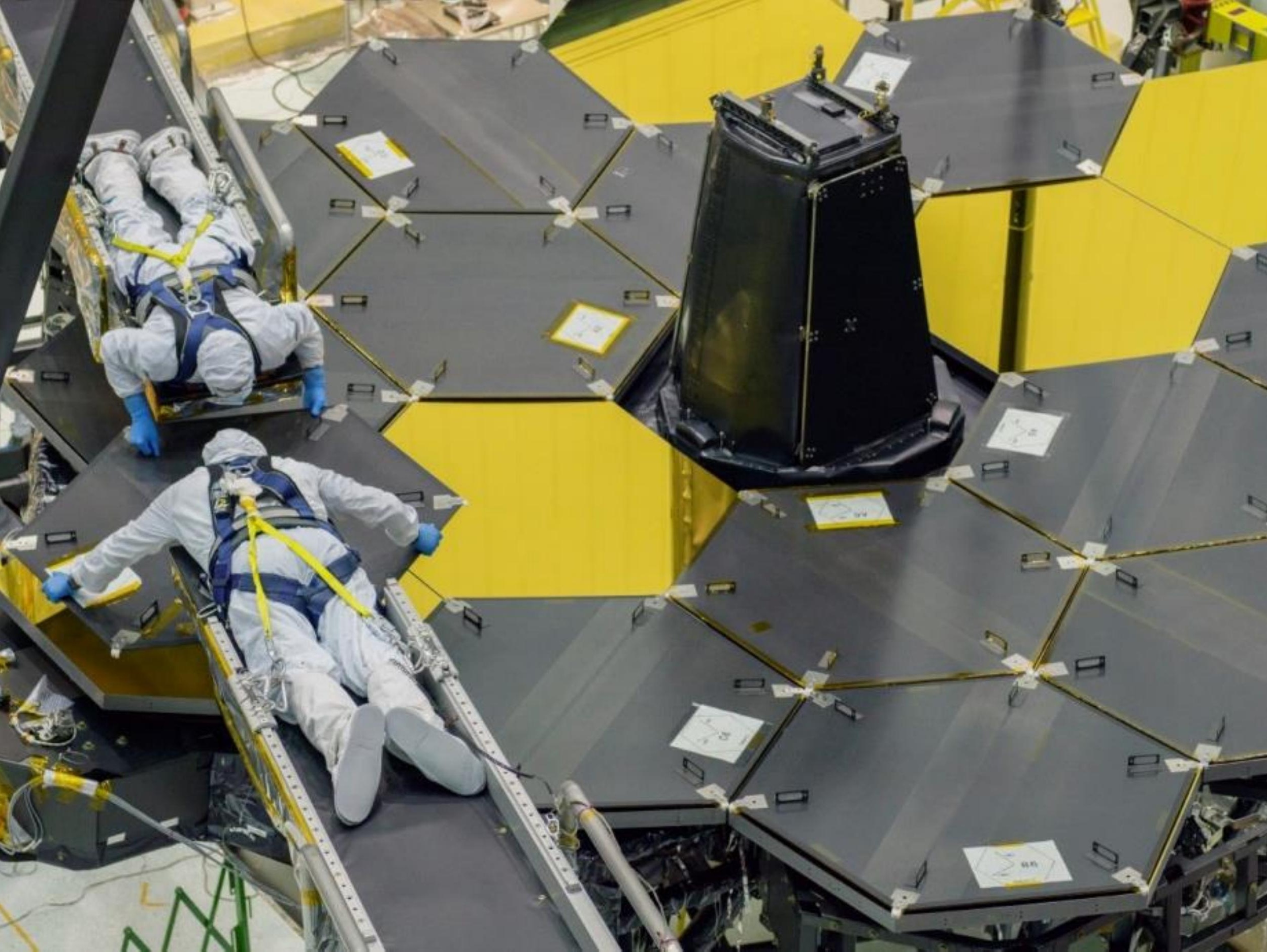




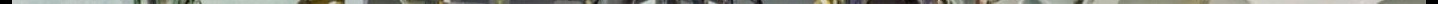




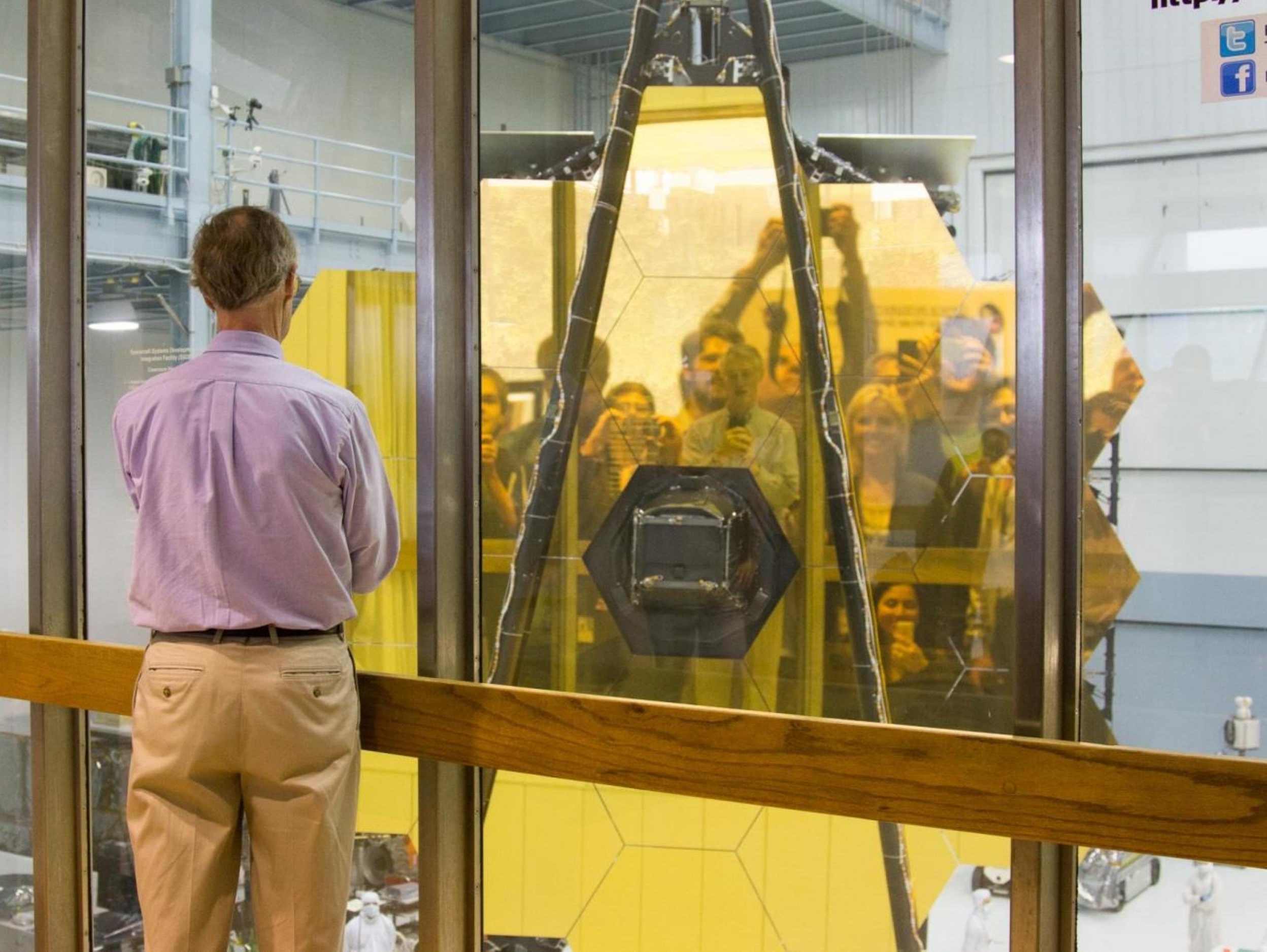




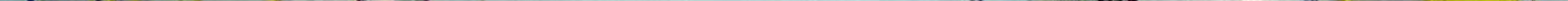




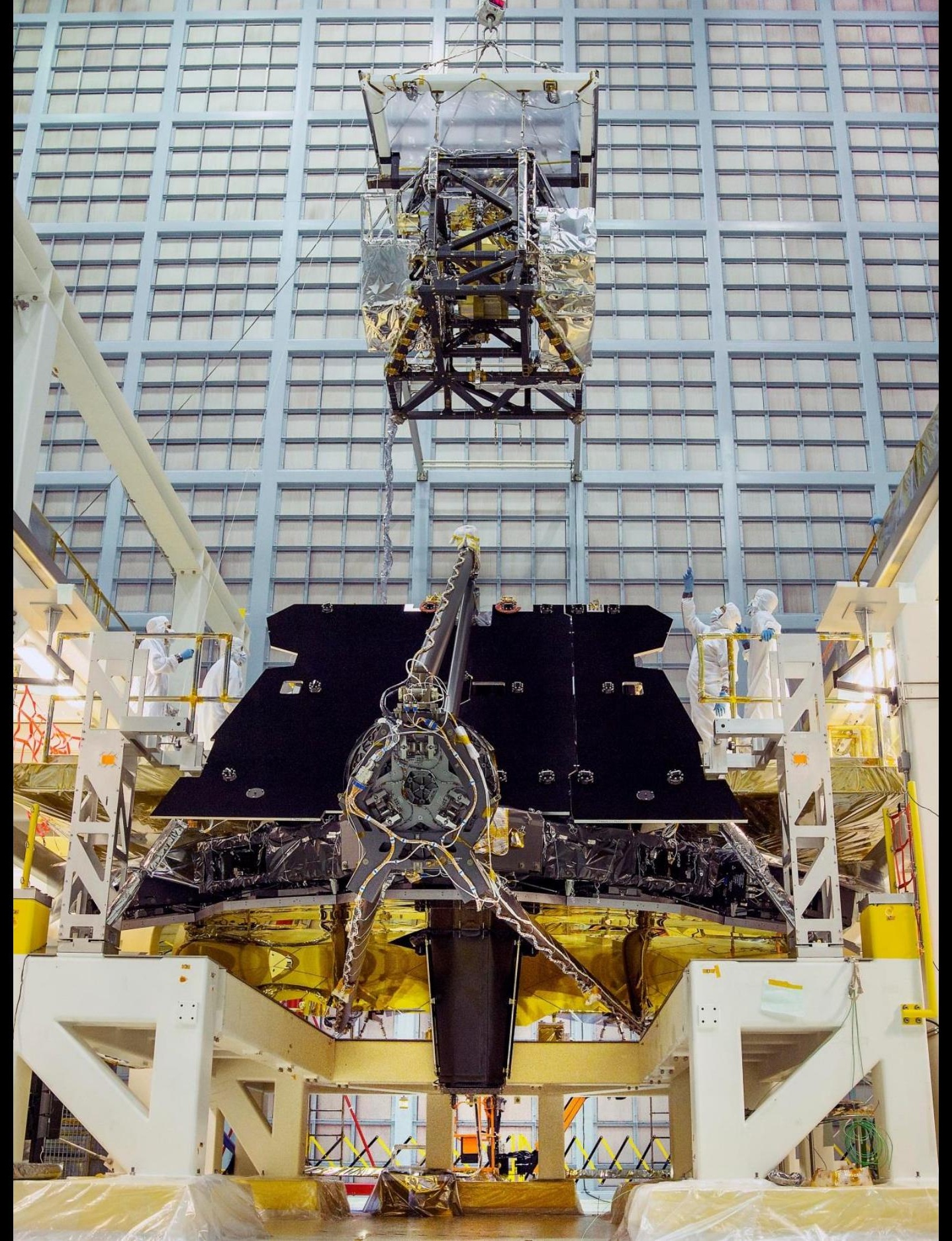




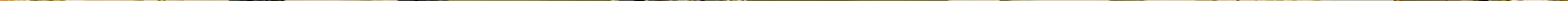




\section{JSC Optical GSE}

- Work continues at JSC to prepare for the OTIS cryo test

- First series of tests were optical based tests

- Check out of the optical ground support equipment

- Increasing complexity for the optical equipment

- Excellent optical results with many lessons learned 


\section{JSC Test Configuration}

NORTHROP GRUMMAN

Ball HNRRIS ATK

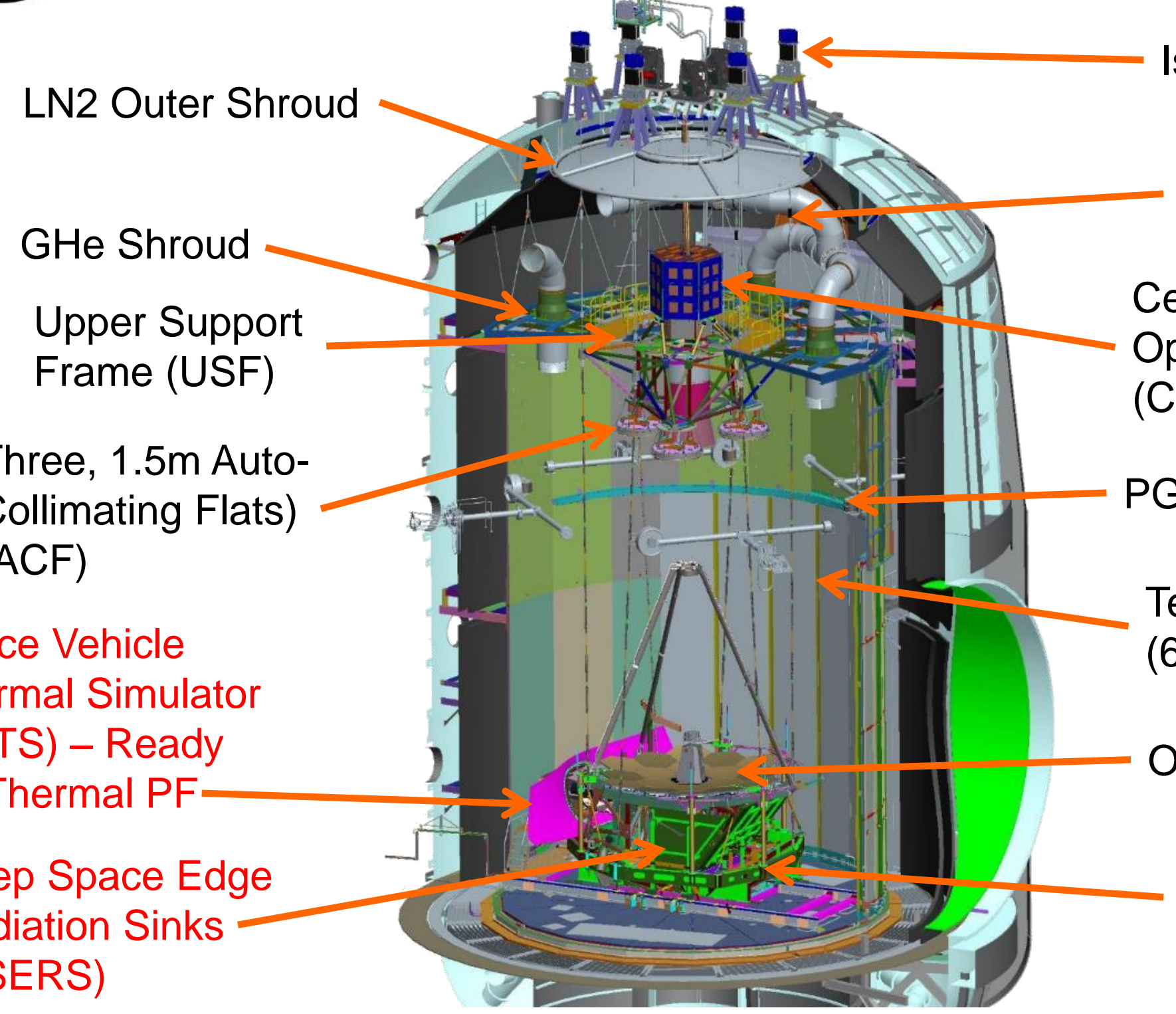

Isolators (6 units)

Downrods (6 units)

Center of Curvature Optical Assembly (COCOA)

Three, 1.5m AutoCollimating Flats) (ACF)

Space Vehicle Thermal Simulator (SVTS) - Ready for Thermal PF-

Deep Space Edge Radiation Sinks (DSERS)

Hardpoint/Offloader Support Structure (HOSS) 


\section{OGSE-1}

Complete

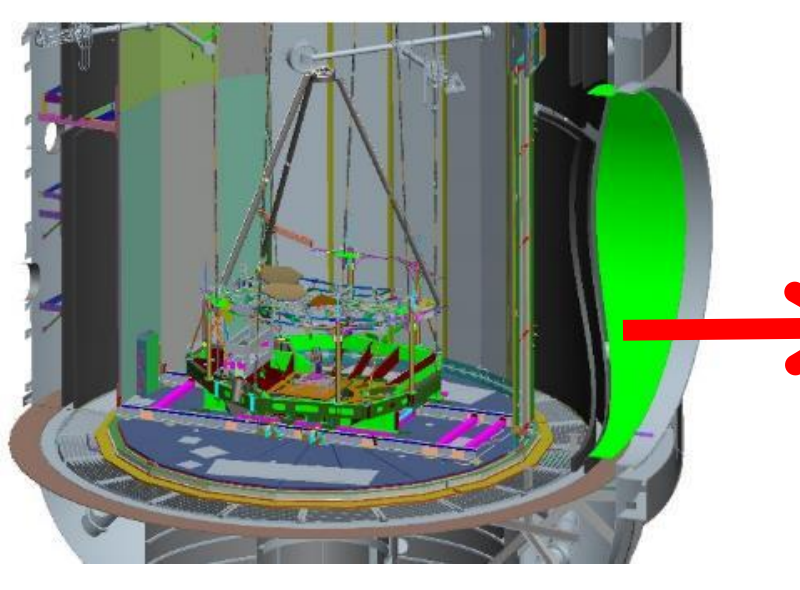

OGSE-2 Complete

\section{Pathfinder Thermal}

PF Updated to be more Flight Like

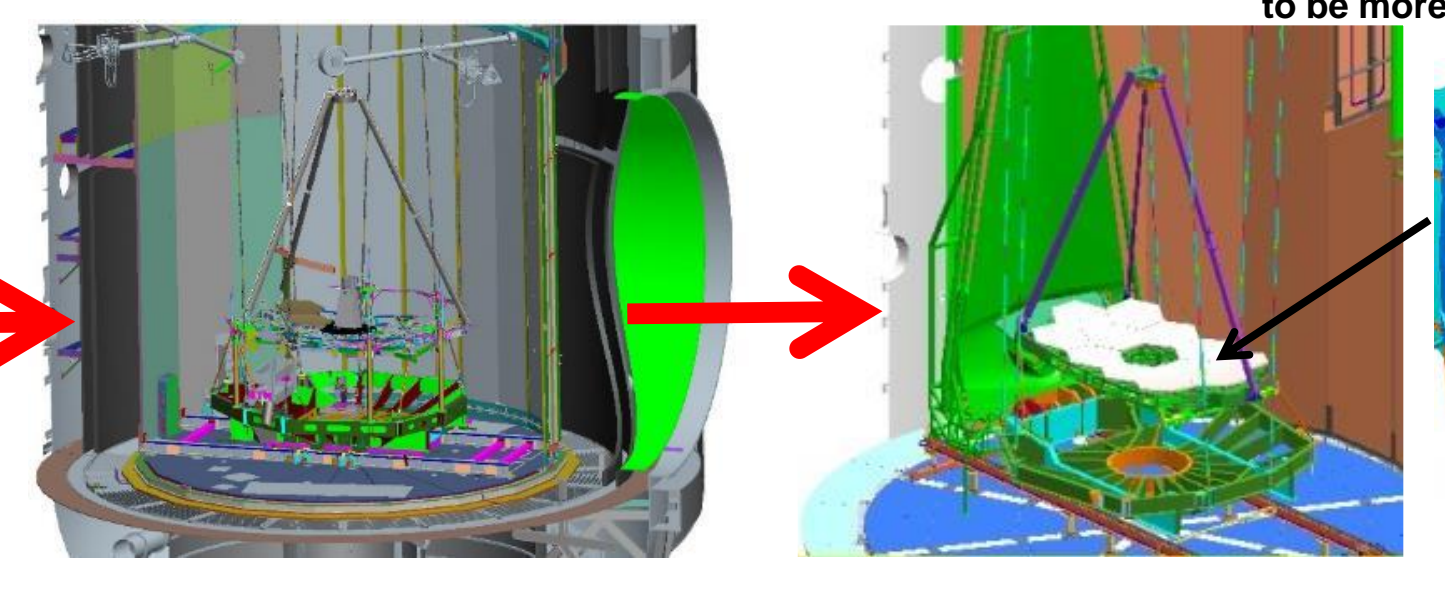

Simulator
- Checkout Optical GSE that has not seen cryo before: CoC test, Hanging Config, Photogrammetry

- No flight hardware except flight spare PMSA/SMA

- Dynamics and Thermal Distortion portion of PF Augmentation occur here
- Checkout Pass and a half test with flight AOS and GSE source plate system

- Uses BIA camera as SI simulator
- Thermal GSE Checkout (includes SVTS)

- Dry run cooldown and warmup

- Will allow risk reduction of some OTE Thermal Balance (design validation off the critical path) 
Chamber Configuration for CCT NASA

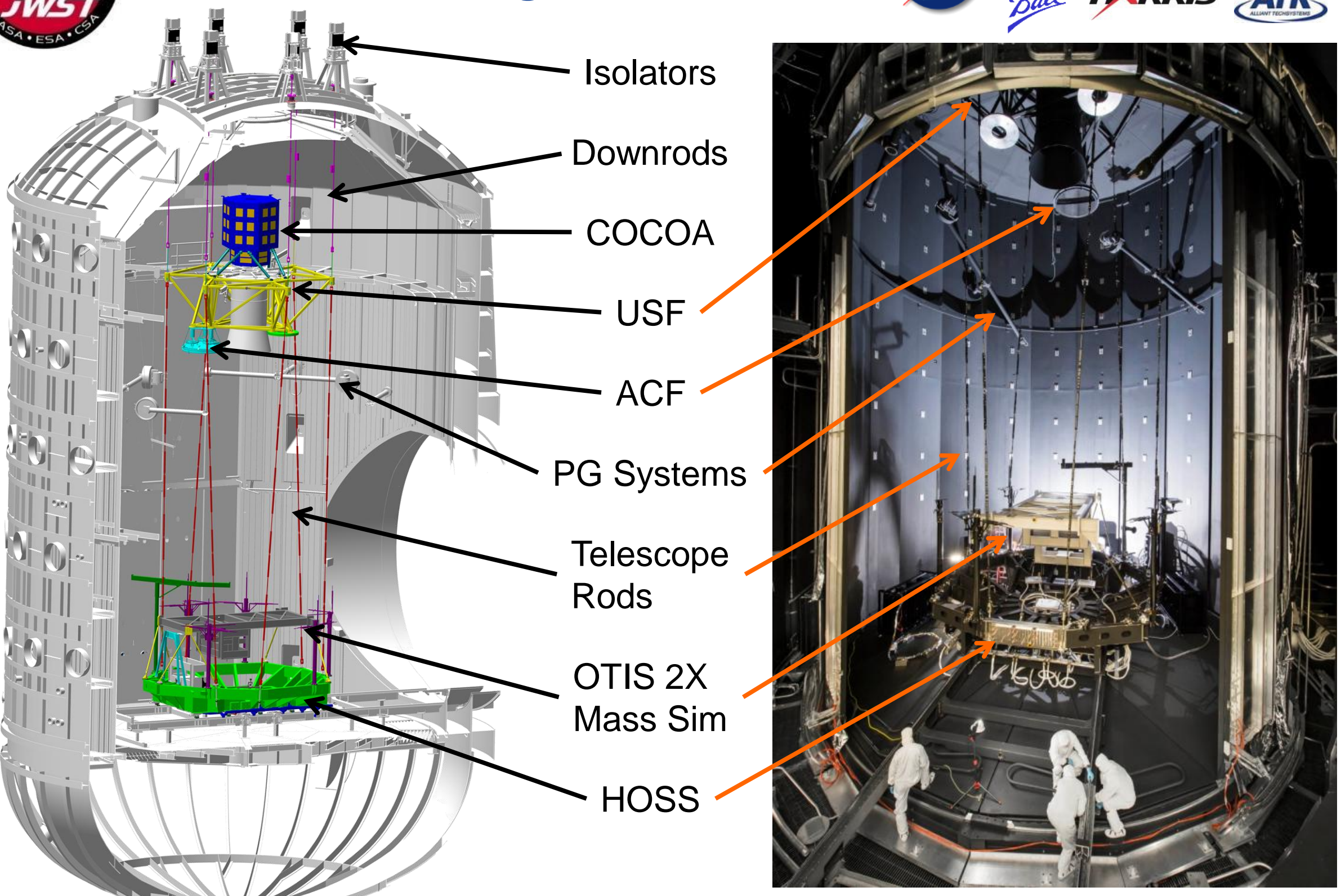




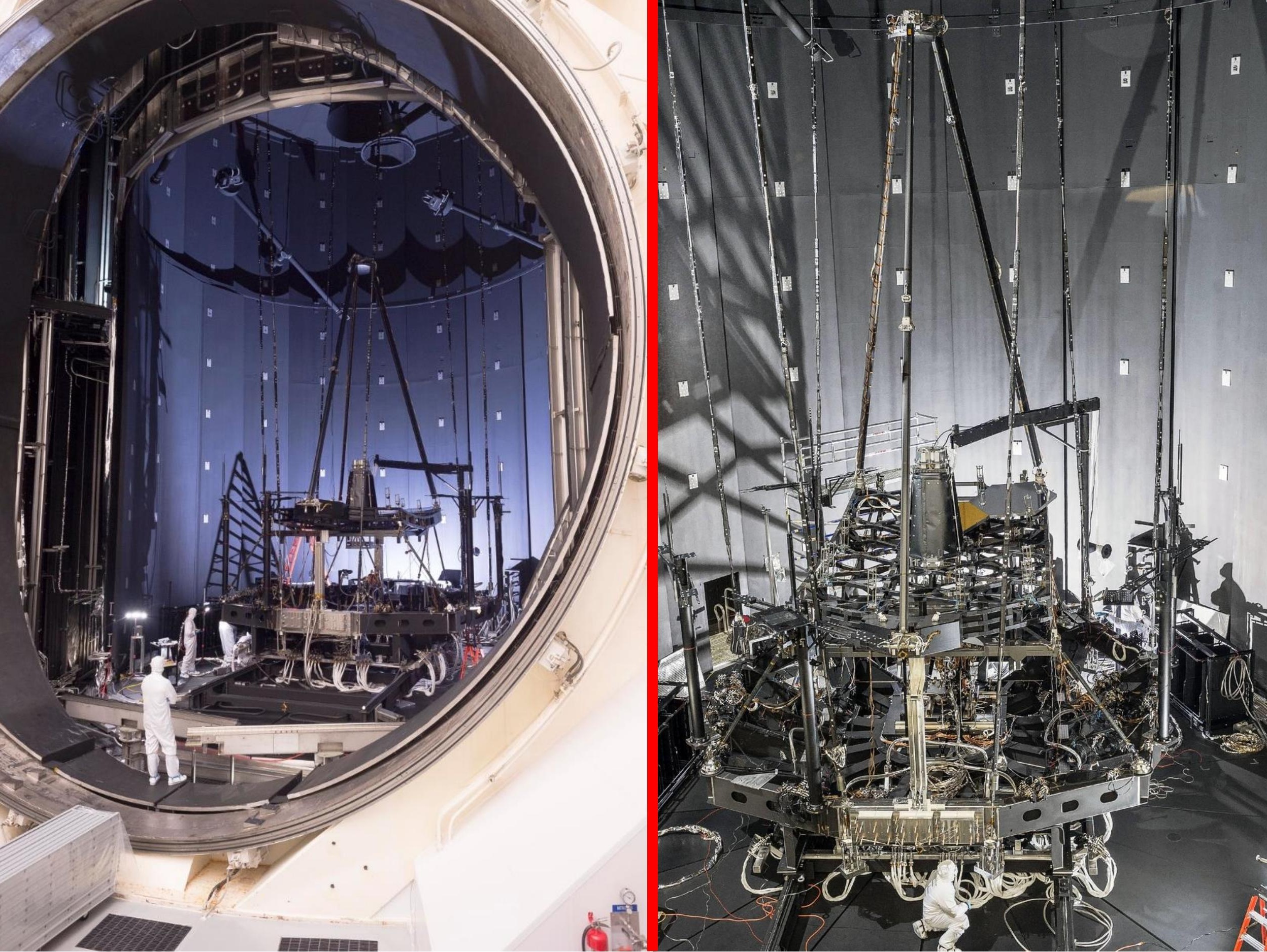




\section{Range of Motion}

- Range of COCOA and ACF actuation systems

\begin{tabular}{|c|c|c|c|c|c|c|}
\hline COCOA & $\begin{array}{c}\text { V1 } \\
(\mathrm{mm})\end{array}$ & $\begin{array}{c}\text { V2 } \\
(\mathrm{mm})\end{array}$ & $\begin{array}{c}\text { V3 } \\
(\mathrm{mm})\end{array}$ & $\begin{array}{c}\text { RV1 } \\
(\mathrm{urad})\end{array}$ & $\begin{array}{c}\text { RV2 } \\
(\text { urad) }\end{array}$ & $\begin{array}{c}\text { RV3 } \\
\text { (urad) }\end{array}$ \\
\hline Range & $+/-24.6$ & $+/-32$ & $+/-32$ & N/A & $+/-5.6$ & $+/-5.6$ \\
\hline $\begin{array}{c}\text { Cryo } \\
\text { Position } \\
\text { (margin) }\end{array}$ & $\begin{array}{c}-7.4 \\
(70 \%)\end{array}$ & $\begin{array}{c}7.2 \\
(76 \%)\end{array}$ & $\begin{array}{c}0.4 \\
(98 \%)\end{array}$ & -0.6 & $\begin{array}{c}0.5 \\
(92 \%)\end{array}$ & $\begin{array}{r}-0.5 \\
(90 \%)\end{array}$ \\
\hline
\end{tabular}

\begin{tabular}{|c|c|c|}
\hline ACF & $\begin{array}{c}\text { Decenter } \\
(\mathbf{m m})\end{array}$ & $\begin{array}{c}\text { Tilt } \\
(\mathrm{mrad})\end{array}$ \\
\hline Range & N/A & 10.8 \\
\hline $\begin{array}{c}\text { Cryo Position } \\
\text { (margin) }\end{array}$ & N/A & $\begin{array}{r}0.15 \\
(99 \%)\end{array}$ \\
\hline
\end{tabular}




\section{Photogrammetry Accuracy}

\begin{tabular}{c|c|c|c|c|}
$\begin{array}{c}\text { Hardware } \\
\text { Component }\end{array}$ & $\begin{array}{c}\text { Measurement } \\
\text { Direction }\end{array}$ & Requirement & $\begin{array}{c}\text { Measured } \\
\text { Value }\end{array}$ & Margin \\
\hline
\end{tabular}

\begin{tabular}{|l|l|l|l|l|}
\hline PM to AOS & Piston & $0.1 \mathrm{~mm}$ & $0.04 \mathrm{~mm}$ & $62 \%$ \\
\hline & Decenter & $0.1 \mathrm{~mm}$ & $0.08 \mathrm{~mm}$ & $16 \%$ \\
\hline & Tilt & $0.15 \mathrm{mrad}$ & $0.09 \mathrm{mrad}$ & $40 \%$ \\
\hline & Clocking & $1.0 \mathrm{mrad}$ & $0.31 \mathrm{mrad}$ & $69 \%$ \\
\hline
\end{tabular}

\begin{tabular}{|l|l|l|l|l|}
\hline SM to AOS & Piston & $0.15 \mathrm{~mm}$ & $0.08 \mathrm{~mm}$ & $43 \%$ \\
\hline & Decenter & $1.25 \mathrm{~mm}$ & $0.65 \mathrm{~mm}$ & $48 \%$ \\
\hline Tilt & $0.335 \mathrm{mrad}$ & $0.27 \mathrm{mrad}$ & $24 \%$ \\
\hline
\end{tabular}




\section{Typical PG Accuracies}

\begin{tabular}{|l|c|c|c|}
\hline & $\mathbf{2 \sigma} \mathbf{~ M 1}(\mathbf{m m})$ & $\mathbf{2 \sigma} \mathbf{M} \mathbf{( m m})$ & $\mathbf{2 \sigma} \mathbf{M} \mathbf{3}(\mathbf{m m})$ \\
\hline ACF & 0.040 & 0.067 & 0.124 \\
\hline SM & 0.034 & 0.020 & 0.058 \\
\hline ASPA Support Arm & 0.047 & 0.021 & 0.022 \\
\hline ASPA & 0.021 & 0.007 & 0.008 \\
\hline AOS Base & 0.020 & 0.015 & 0.013 \\
\hline PM & 0.023 & 0.010 & 0.014 \\
\hline Strut Base & 0.116 & 0.024 & 0.053 \\
\hline
\end{tabular}




\section{PMSA Figure Error}

Measured (165 nm-rms) Model Predict (161 nm-rms) Difference (31 nm-rms)

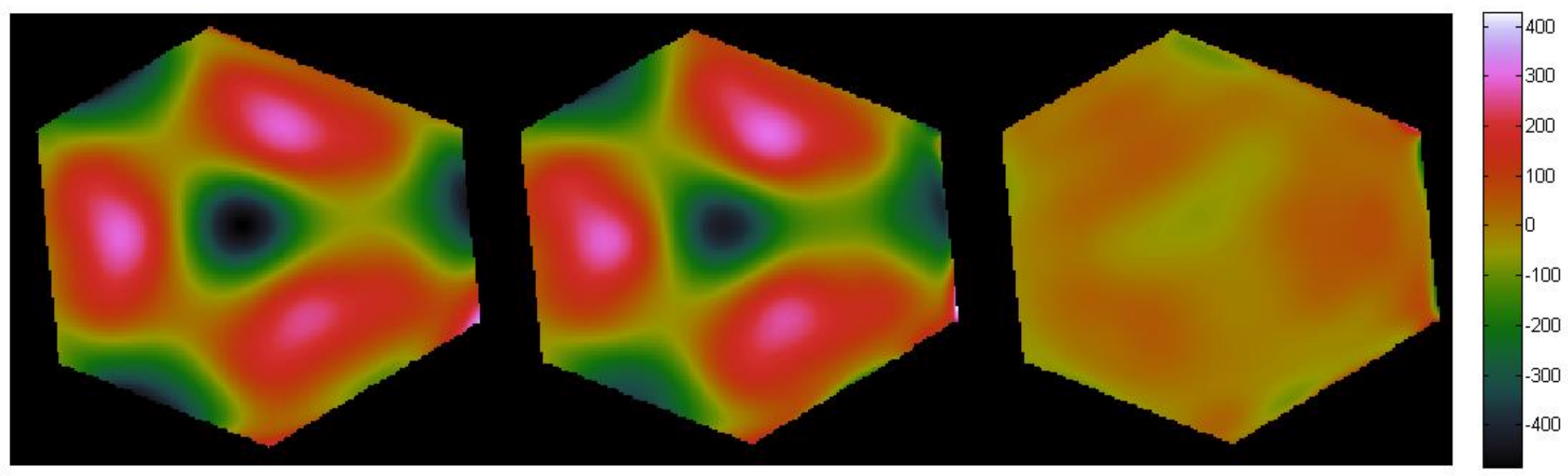




\section{Inward Source Light Path}

ASPA Inward

Source

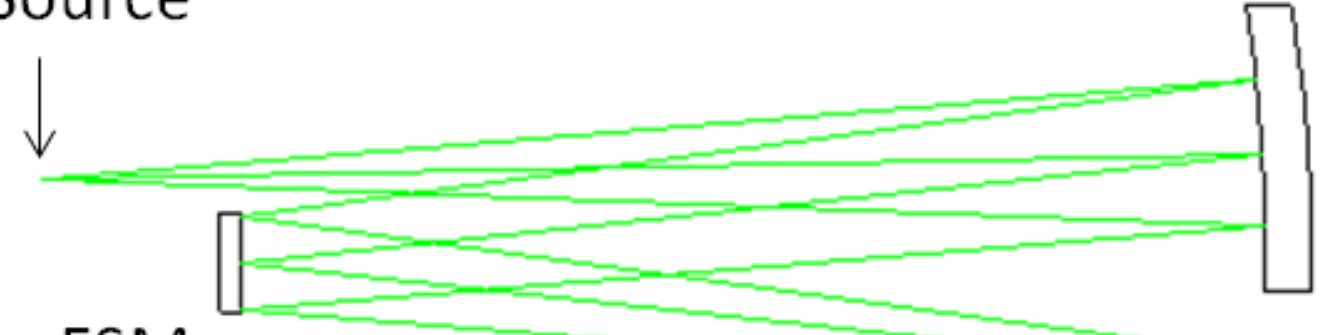

FSM

TMA

Image of ASPA Inward

Source $\downarrow$ 


\section{ASPA Image Location}

\begin{tabular}{|c|c|c|c|c|}
\hline \multirow[b]{2}{*}{ Instrument FOV } & \multirow{2}{*}{$\begin{array}{l}\text { ASPA Source } \\
\text { Designation }\end{array}$} & \multicolumn{3}{|c|}{$\begin{array}{c}\text { Difference between Best Image Location and } \\
\text { Predicted Location }\end{array}$} \\
\hline & & $\mathrm{dM1}(\mathrm{mm})$ & $\mathrm{dM} 2(\mathrm{~mm})$ & dM3 (mm) \\
\hline NIRCamB & $\mathrm{I}-1$ & 0.987 & 0.137 & -0.120 \\
\hline NIRCamB & $1-3$ & 0.578 & 0.055 & -0.395 \\
\hline NIRCamB & $1-4$ & 0.792 & 0.168 & 0.127 \\
\hline NIRCamA & $1-5$ & 0.784 & 0.327 & -0.151 \\
\hline NIRCamA & $1-6$ & 0.749 & -0.176 & -0.192 \\
\hline FGS1 & $1-7$ & 0.579 & 0.030 & -0.204 \\
\hline FGS1 & $1-8$ & 0.531 & 0.013 & -0.176 \\
\hline FGS1 & $1-9$ & 0.167 & 0.099 & -0.215 \\
\hline FGS2 & $\mathrm{I}-11$ & 0.946 & 0.060 & -0.092 \\
\hline FGS2 & $\mathrm{I}-13$ & 0.870 & 0.069 & -0.094 \\
\hline NIRISS & $\mathrm{I}-15$ & -0.129 & 0.051 & -0.267 \\
\hline NIRISS & $\mathrm{I}-16$ & 0.084 & 0.074 & -0.188 \\
\hline MIRI & $1-23$ & 0.013 & 0.195 & 0.135 \\
\hline MIRI & $1-24$ & -0.217 & 0.108 & -0.385 \\
\hline NIRSPEC & $1-25$ & 0.265 & 0.102 & -0.191 \\
\hline NIRSPEC & $1-26$ & -0.035 & 0.094 & -0.247 \\
\hline \multirow{3}{*}{\multicolumn{2}{|c|}{$\begin{array}{l}\text { Average } \\
\text { Standard Deviation } \\
\text { Range }\end{array}$}} & 0.435 & 0.088 & -0.166 \\
\hline & & 0.410 & 0.103 & 0.144 \\
\hline & & 1.204 & 0.503 & 0.530 \\
\hline
\end{tabular}




\section{Hartmann Test Sensivity}

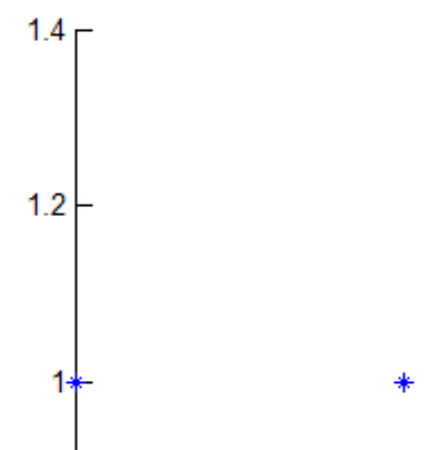

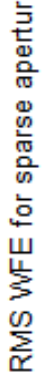

峞 0.6

.

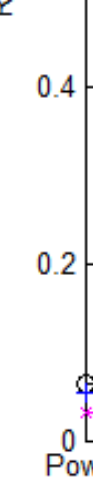

$$
\text { Power }
$$

Astig

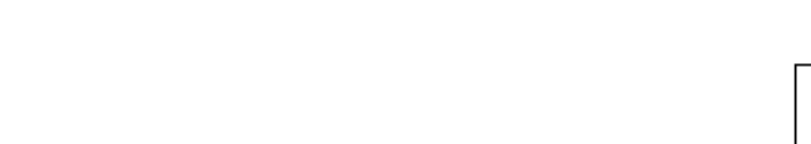

\section{Ball HARRIS ATK}

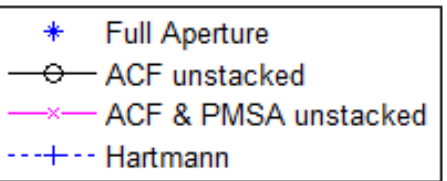

\section{Baseline}

Hartmann 


\section{Fid Light Bar Performance}
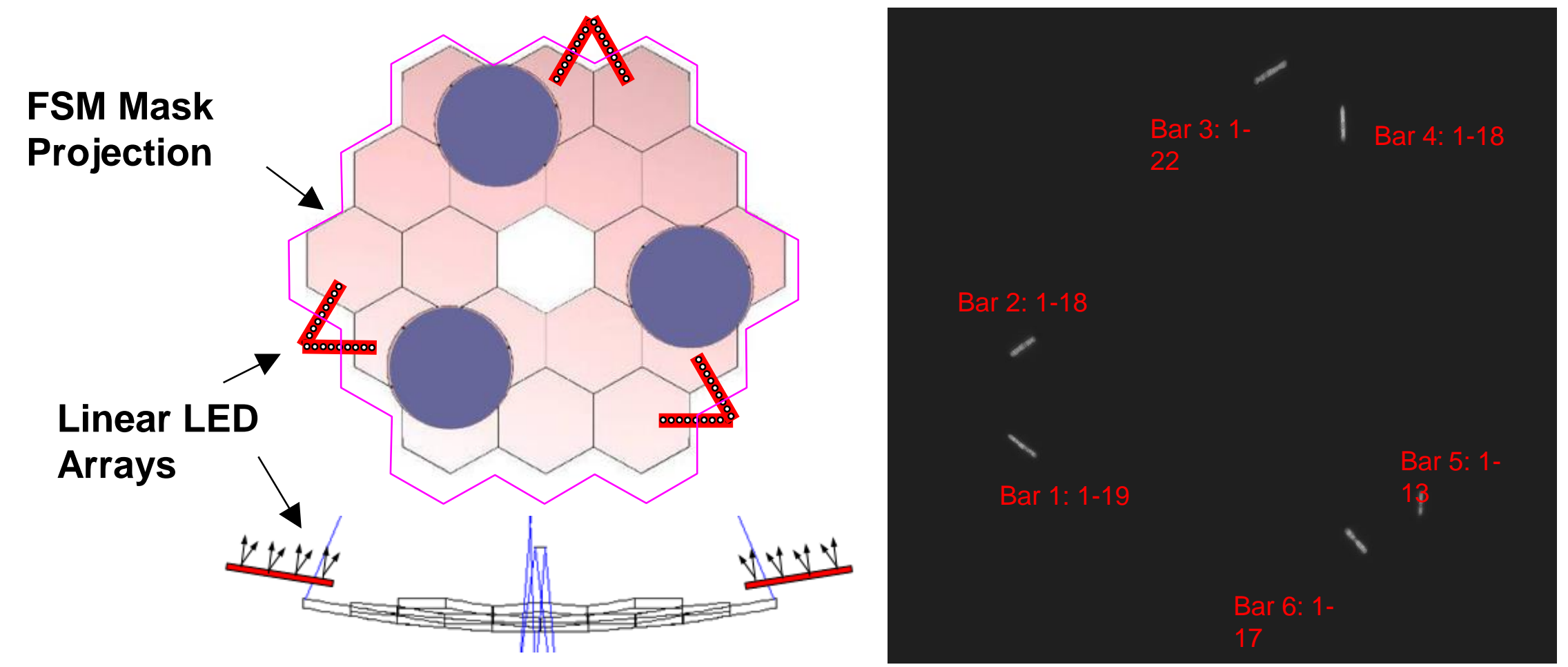

\begin{tabular}{|l|c|c|c|c|c|c|}
\hline Bar & $\mathbf{1}$ & $\mathbf{2}$ & $\mathbf{3}$ & $\mathbf{4}$ & $\mathbf{5}$ & $\mathbf{6}$ \\
\hline Nominal Last visible LED & 20 & 18 & 21 & 17 & 13 & 16 \\
\hline Actual last visible LED & 19 & 18 & 22 & 18 & 13 & 17 \\
\hline
\end{tabular}


Shadowgram Test

NORTHROP GRUMMAN

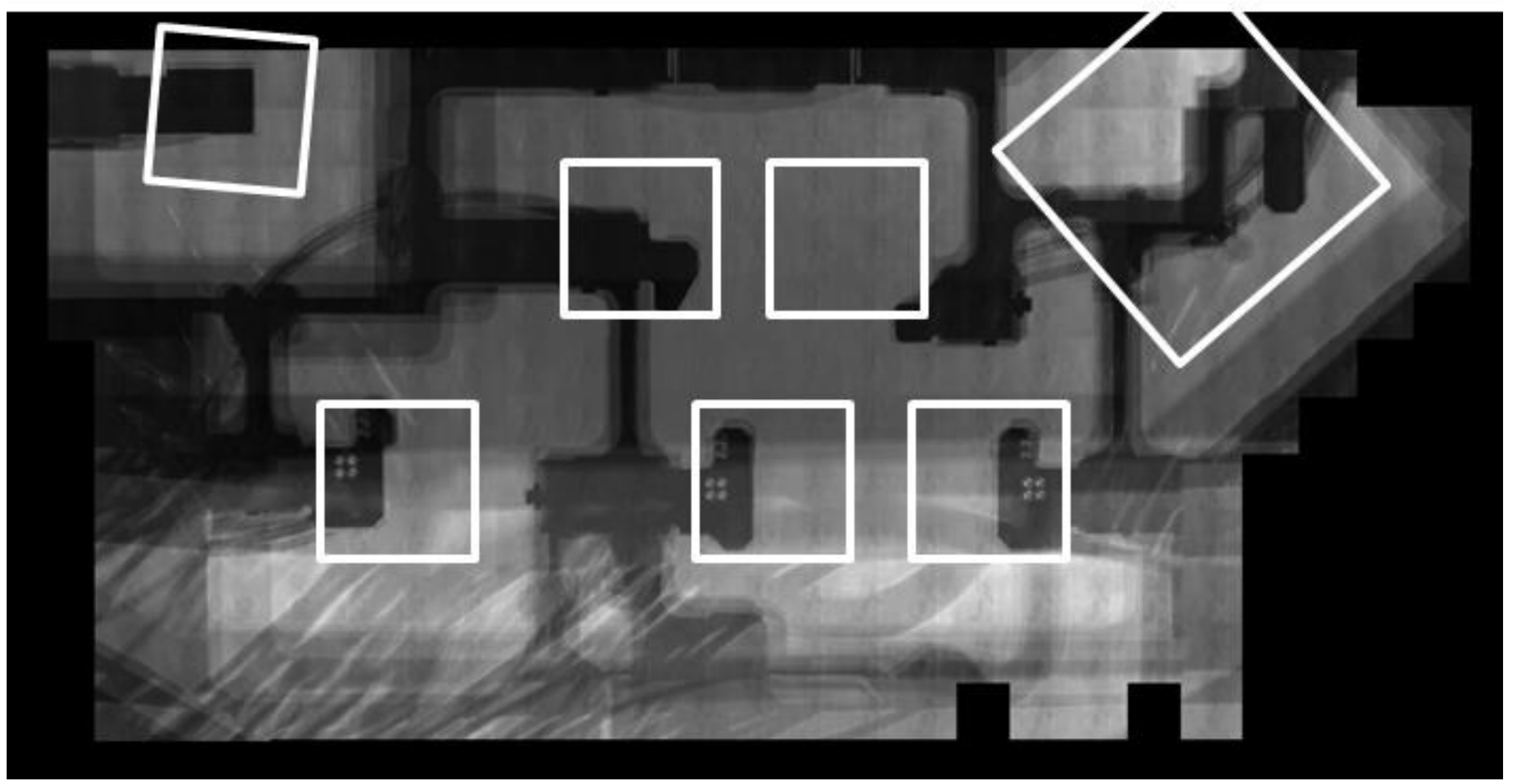




\section{Thermal Pathfinder (TPF) Test}

- Final test prior to OTIS is thermally focused

- How does the system cool down with an OTIS simulator

- Incorporation of all the thermal hardware

- DSERS

- SVTS

- Zero-Q heaters

- Actively cooled ACF's due to "no He gas" for the OTIS test 
Dit 111

14

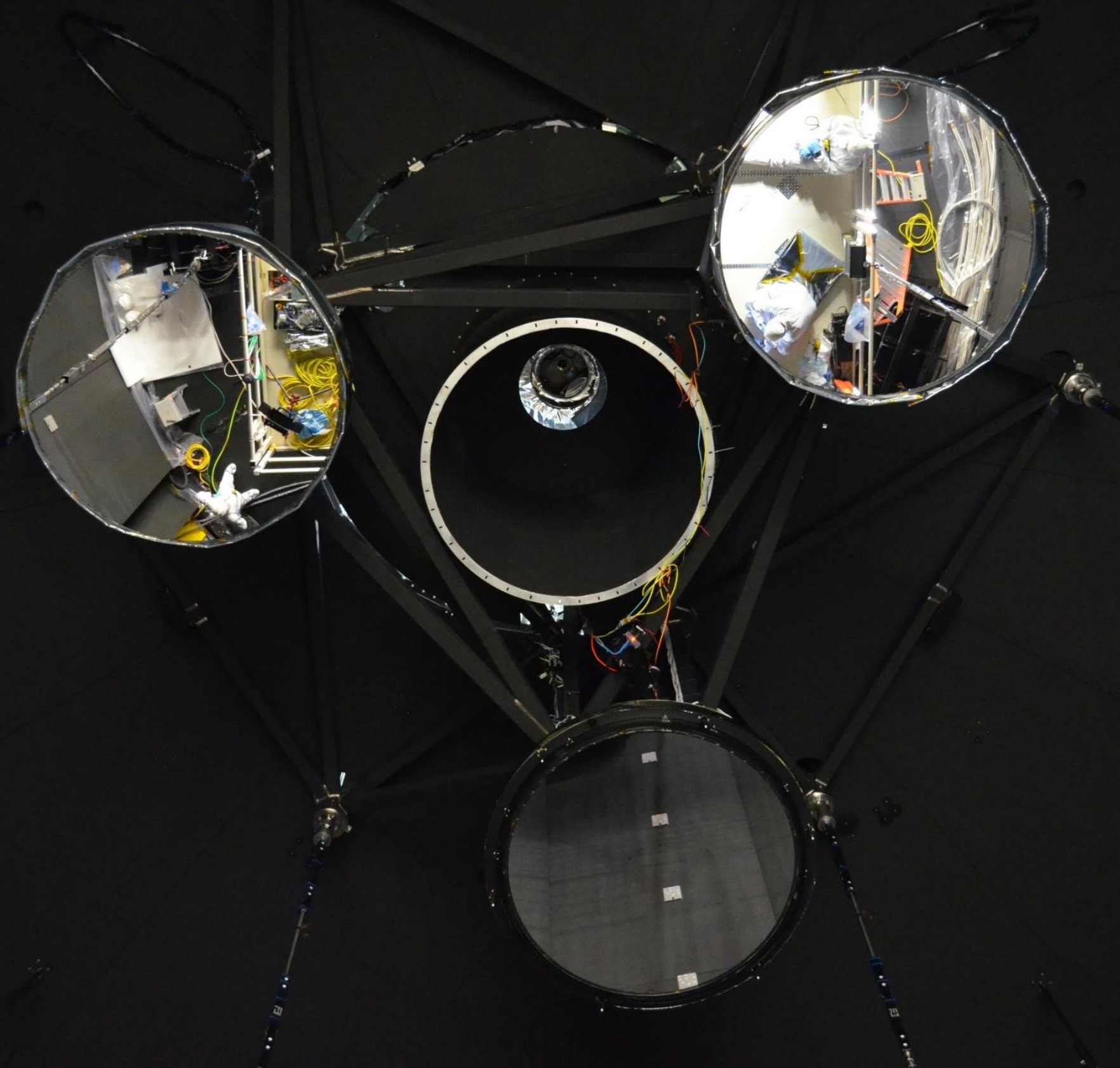




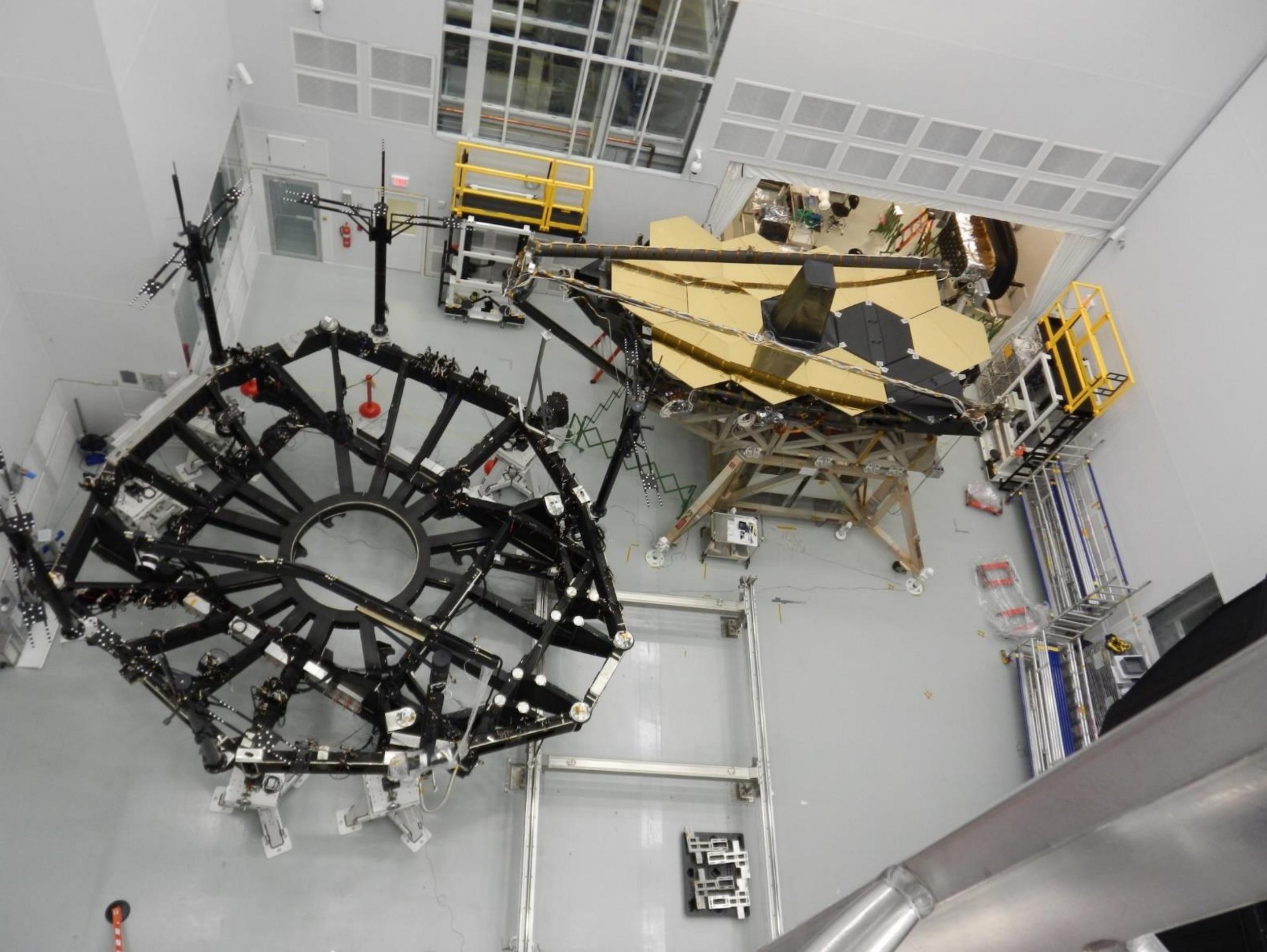




\section{HOSS Thermal Configuration}

NORTHROP GRUMMAN

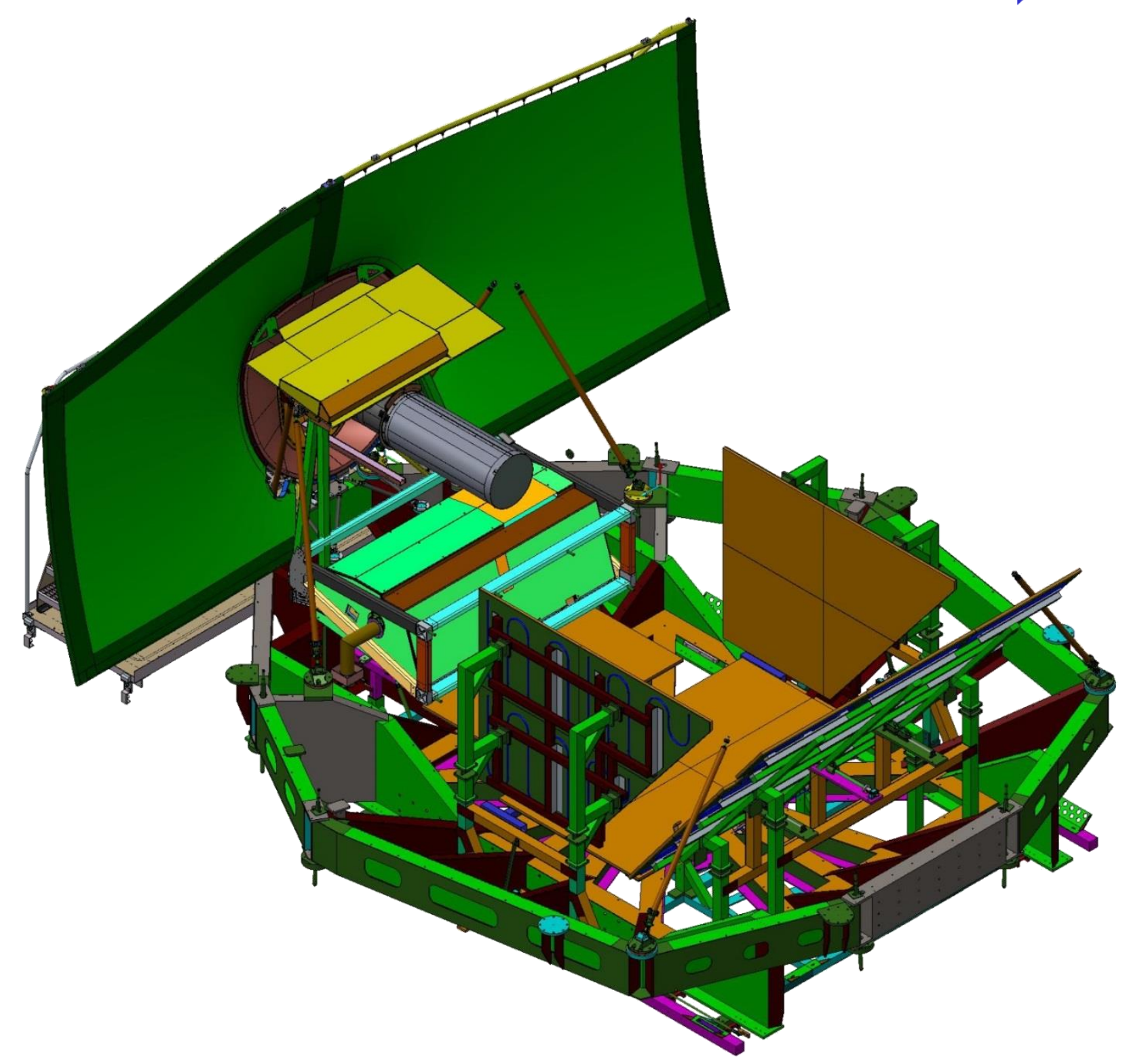

Ball HYRRIS ATK 


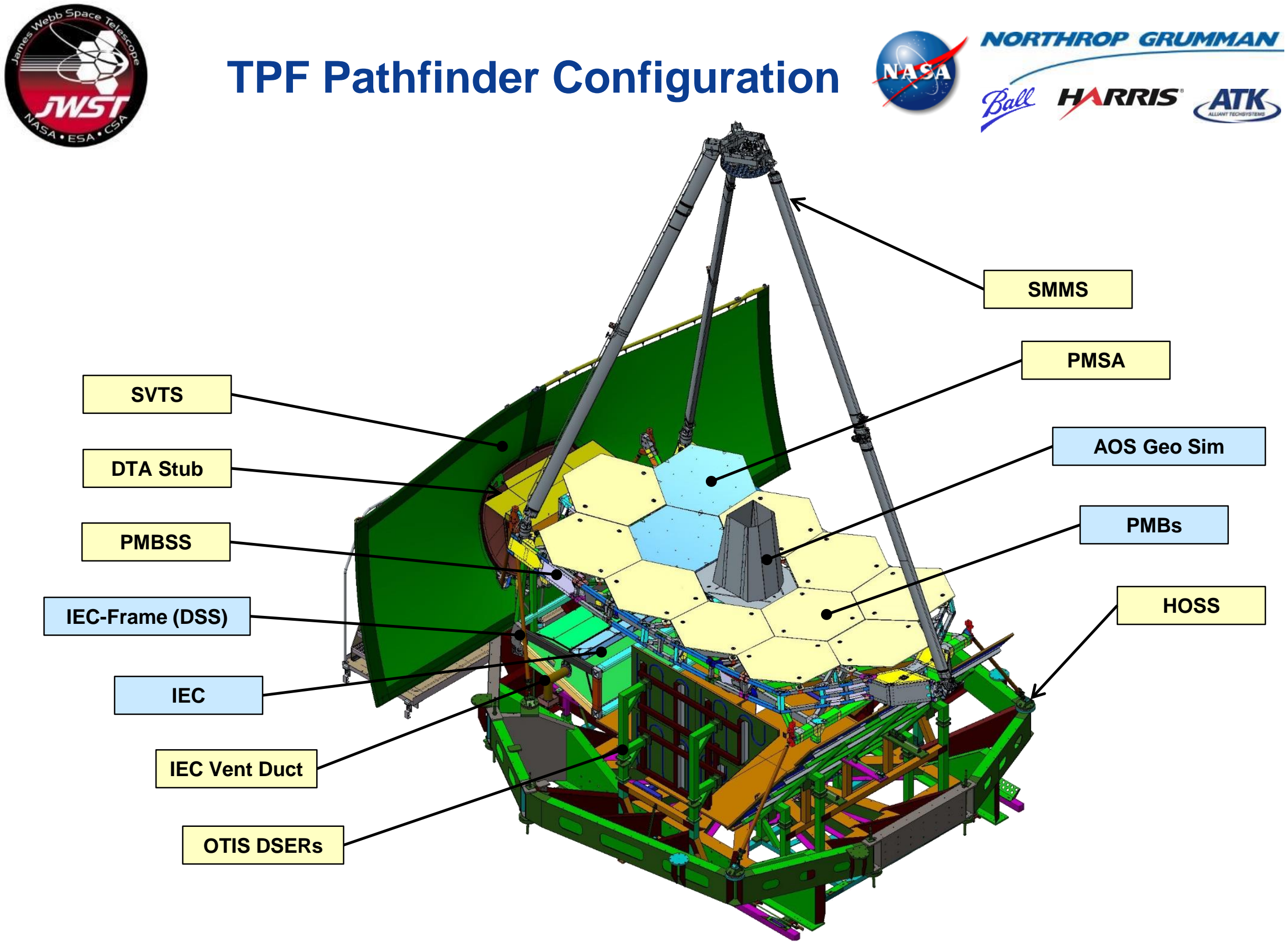




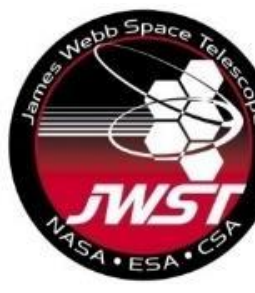

SVTS Hub and Rim

NORTHROP GRUMMAN

Ball HIRRIS ATK

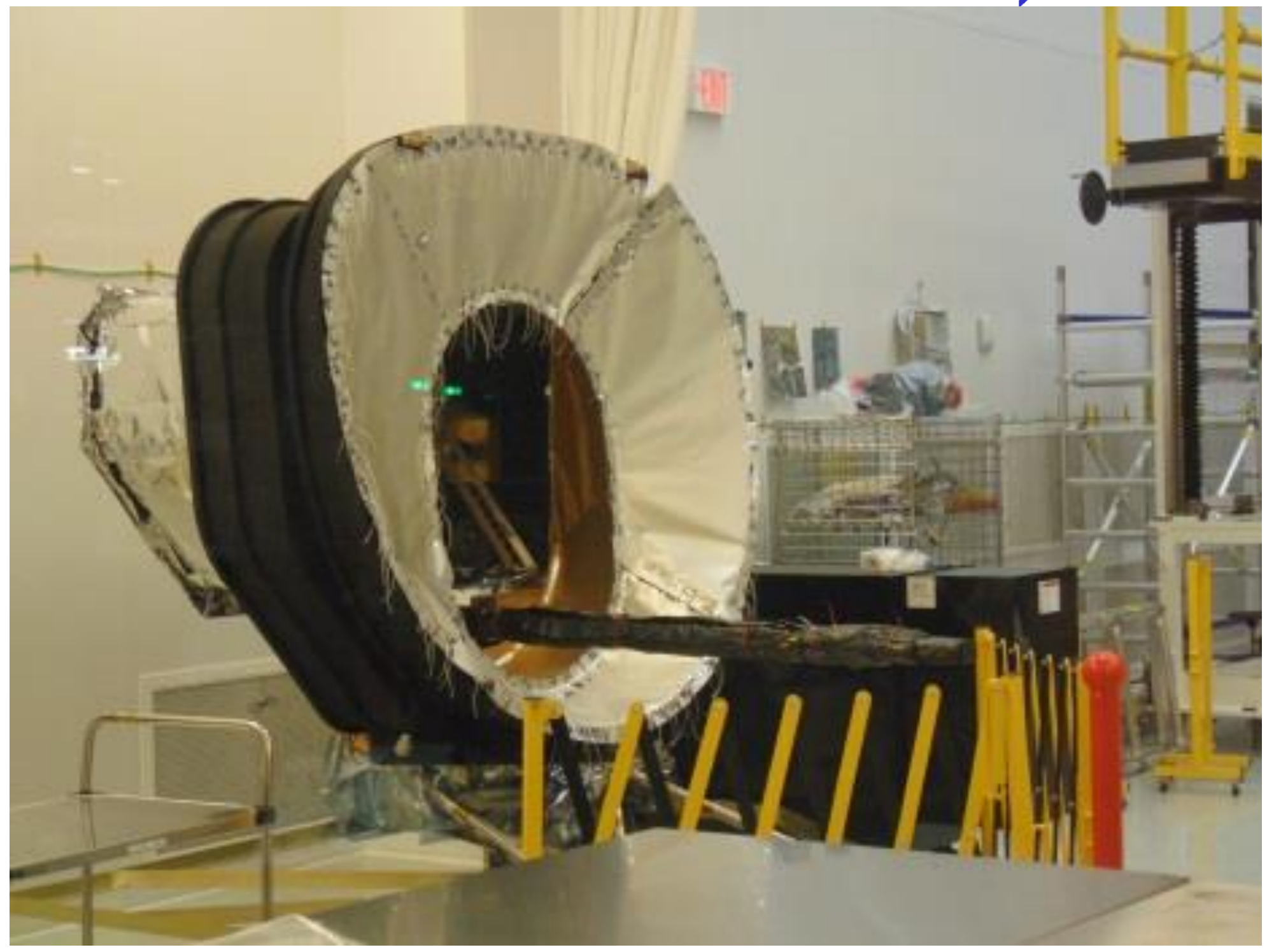




\section{Summary}

- The first of three Optical Ground Support Equipment and Thermal Tests is complete

- Test results have been excellent

- COCOA works as designed

- The two PMSA's were phased

- PG system is fully operational

- Isolation system worked as designed

- Short during cryo temperatures identified and will be corrected prior to OGSE\#2

- Pathfinder has been very important and enables the flight program

- Provides critical experience in preparation for the critical path flight program

- Well worth the investment by the program 